\title{
Qualitative and quantitative evaluation of dynamic changes in non-culprit coronary atherosclerotic lesion morphology: a longitudinal OCT study
}

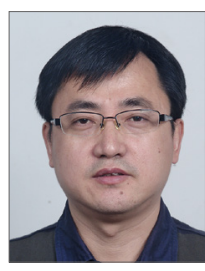

Bu-Chun Zhang ${ }^{1,2}, \mathrm{MD}$; Antonios Karanasos ${ }^{1,3 *}, \mathrm{MD}, \mathrm{PhD}$;

Muthukaruppan Gnanadesigan ${ }^{4}, \mathrm{PhD}$; Johannes van der Sijde ${ }^{1}, \mathrm{MD}$;

Nienke van Ditzhuijzen ${ }^{1}$, MSc; Karen Witberg ${ }^{1}$, RN; Jurgen Ligthart ${ }^{1}$, BSc;

Roberto Diletti ${ }^{1}, \mathrm{MD}, \mathrm{PhD}$; Robert-Jan van Geuns ${ }^{1}, \mathrm{MD}$, PhD; Jouke Dijkstra ${ }^{5}, \mathrm{PhD}$;

Felix Zijlstra ${ }^{1}, \mathrm{MD}$, PhD; Gijs van Soest ${ }^{4}, \mathrm{PhD}$; Evelyn Regar, ${ }^{1,6}, \mathrm{MD}, \mathrm{PhD}$

1. Department of Cardiology, Erasmus Medical Center, Rotterdam, the Netherlands; 2. The Affiliated Hospital of Xuzhou Medical University, Jiangsu, China; 3. Department of Cardiology, Hippokration Hospital, Athens, Greece; 4. Department of Biomedical Engineering, Erasmus Medical Center, Rotterdam, the Netherlands; 5. Department of Radiology, Leiden University Medical Center, Leiden, the Netherlands; 6. Department of Cardiovascular Surgery, University Hospital Zurich, Zurich, Switzerland

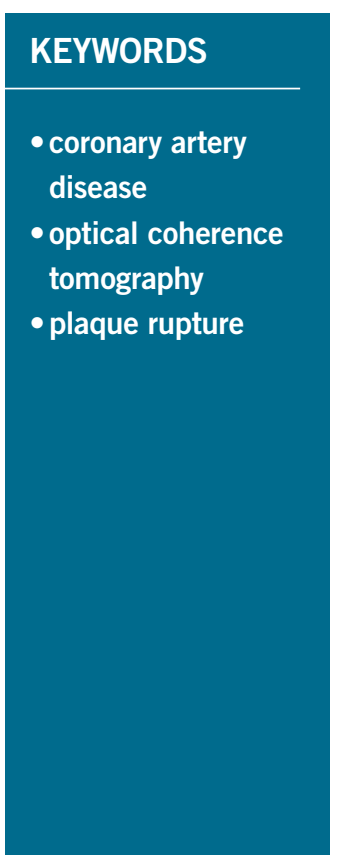

\begin{abstract}
Aims: There is limited in vivo evidence regarding the temporal evolution of non-culprit coronary plaque morphology. We aimed to evaluate changes in non-culprit plaque morphology over time by optical coherence tomography (OCT).

Methods and results: Seventy-two (72) patients with 257 non-culprit segments with serial OCT studies were analysed. Non-culprit $5 \mathrm{~mm}$-long coronary segments from the same imaged region were matched between baseline and follow-up. OCT plaque characterisation including automated attenuation analysis was performed, and changes over a median follow-up of 6.2 months were evaluated. On segment level, lumen area decreased from baseline to follow-up, whereas fibrous cap thickness increased. Similarly, plaque attenuation indices at follow-up were significantly decreased. Minimal cap thickness per patient did not change. In $68.5 \%$ of segments, plaque morphology did not change. Favourable change was observed in $18.4 \%$ of segments and unfavourable in $12.9 \%$. There were no robust clinical predictors of change in plaque morphology. Attenuation analysis supported the qualitative characterisation, showing significantly different attenuation between different plaque types.
\end{abstract}

Conclusions: In non-culprit coronary segments of patients with coronary artery disease under standard medical therapy, segment-level but not patient-level minimum fibrous cap thickness increases over time, with observations of both favourable and unfavourable changes in individual segments.

\footnotetext{
*Corresponding author: Hippokration Hospital, Omirou 19, 15344 Gerakas, Greece. 


\section{Abbreviations}

CAD coronary artery disease

IPA index of plaque attenuation

MLA minimal lumen area

NSTEMI non-ST-elevation myocardial infarction

OCT optical coherence tomography

OR odds ratio

RCI relative calcium index

RLCI relative lipid core index

ROI region of interest

TCFA thin-cap fibroatheroma

VH-IVUS virtual histology intravascular ultrasound

\section{Introduction}

Post-mortem evidence suggests that plaque morphology is implicated in plaque destabilisation ${ }^{1}$. However, prospective studies have only demonstrated a modest prognostic significance of plaque morphology for future events ${ }^{2}$. In order to enhance this prognostic capability, in vivo morphological characteristics associated with plaque progression should be further defined and morphological changes over time documented. Optical coherence tomography (OCT) allows a comprehensive morphological plaque characterisation $^{3}$, further corroborated by automated plaque characterisation ${ }^{4}$.

We aimed to evaluate changes in plaque morphology of nonculprit lesions over time by OCT using a qualitative and a quantitative approach, and identify predictive factors.

Editorial, see page 2105

\section{Methods}

\section{STUDY PROTOCOL}

The OCT database of the Erasmus MC was screened for patients undergoing serial OCT of the same single vessel within three months. Indications for repeat catheterisation included new symptoms, staged procedures or study patients. Patients in whom the same non-culprit native segment was visualised at both time points were included. The pullbacks were matched using landmarks and synchronised by an automated algorithm. OCT analysis was then performed in the matched vessel region, and changes were recorded. Analysis was performed on patient level (vessel level; all matched segments from a single vessel) and on segment level (the imaged region was divided in equal subsegments of $5 \mathrm{~mm}$ ). Additionally, quantitative plaque characterisation by automated attenuation analysis was performed. Demographic and clinical data were collected retrospectively. Informed consent was acquired from all patients and the study complied with the Declaration of Helsinki.

\section{STUDY POPULATION}

All patients $>18$ years old with serial OCT examination of the same vessel for clinical or research purposes at the Erasmus MC between 1 January 2009 and 31 August 2014, and with $>3$ months interval between the two examinations were screened. Patients in whom the same non-culprit native coronary segment $\geq 5 \mathrm{~mm}$ in length and located $>5 \mathrm{~mm}$ beyond the edge segment of an implanted device was identified in both pullbacks were finally included. Exclusion criteria were invasive treatment of a non-culprit lesion between baseline and follow-up, poor image quality, and saphenous vein graft lesions.

\section{OCT IMAGE ACQUISITION}

OCT was performed according to our catheterisation laboratory practice using the C7 OPTISTM imaging system and Dragonfly ${ }^{\mathrm{TM}}$ catheter (LightLab/St. Jude, St. Paul, MN, USA), or the Lunawave ${ }^{\circledR}$ system with the FastView ${ }^{\circledR}$ catheter (Terumo Corp., Tokyo, Japan) ${ }^{5}$.

\section{OCT ANALYSIS}

\section{REGION OF INTEREST (ROI) AND SEGMENT MATCHING}

Matching was performed using dedicated software (QCU-CMS; LKEB, Leiden, the Netherlands). Baseline and follow-up pullbacks were simultaneously reviewed for identification of a matched native coronary segment. Segment identification was based on anatomic (side branches) or device-specific (stent edges) landmarks. As longitudinal catheter motion does not allow precise co-registration by a single landmark and length information ${ }^{6}$, multiple landmarks were selected, including stent edges and side branches to improve precision, and segment co-registration was performed. This allowed synchronised scrolling, used to identify frame numbers corresponding to the same segment in the two pullbacks, thus comprising the ROI. For segment-level analysis, the baseline ROI was divided into subsegments of an arbitrary length of $5 \mathrm{~mm}$, allowing minimisation of potential co-registration errors by decreasing the segment-tolandmark ratio. The segments were matched after identification of corresponding frames by synchronised scrolling.

\section{LUMEN MEASUREMENTS}

Lumen measurements were performed every $1 \mathrm{~mm}$, and mean and minimal lumen area were calculated for patient and segment level.

\section{PLAQUE ANALYSIS}

Plaque morphology was assessed for patient and segment level at $1 \mathrm{~mm}$ intervals within the ROI, using commonly accepted definitions $^{7}$. Lipid core and calcium were recorded and their arc (in ${ }^{\circ}$ ) was measured. Measurements included mean and maximum lipid core and calcium arc, and also relative volumetric indices of lipid core and calcium content.

The relative lipid core index (RLCI) was calculated as: RLCI $=($ mean lipid core arc $\times$ lipid core length $) /(360 \times$ analysed length $)$.

Similarly, the relative calcium index (RCI) was calculated as: $\mathrm{RCI}=($ mean calcium arc $\times$ calcium length $) /(360 \times$ analysed length $)$.

The cap overlying lipid core was measured over its entire surface by a semi-automated algorithm, with subsequent manual corrections if necessary ${ }^{8}$. The minimum and average cap thicknesses per patient and per segment were then calculated ${ }^{7}$.

\section{INTER-STUDY VARIABILITY}

As analysis of matched segments might contain bias of global measurements $^{6}$, inter-study variability was calculated ${ }^{9}$. In 10 random patients, five at baseline and five at follow-up, a second pullback 
of the same vessel at the same interval was analysed. Matching was performed as described above, and OCT analysis was performed. Bias and $95 \%$ limits of agreement for OCT metrics were calculated, and only differences from baseline to follow-up beyond the limits of agreement were considered relevant. Intra- and inter-observer variability was assessed in the same segments .

\section{HIERARCHICAL PLAQUE CHARACTERISATION}

Plaque characterisation was performed for patient and segment level using a hierarchical definition (Figure 1).

\section{ATTENUATION ANALYSIS}

We applied quantitative plaque characterisation by attenuation analysis ${ }^{4}$. The attenuation coefficient is a robust tissue optical parameter, increased in lipid core and tissue macrophages, compared to calcium and fibrous tissue ${ }^{10}$. Values of attenuation coefficient for the entire perimeter of each cross-section for the entire studied segment were graphically plotted in a longitudinal attenuation vessel map. This display depicts the maximum attenuation between lumen and intima-media border ${ }^{11}$. Thereby the index of plaque attenuation (IPA) is derived. IPA is a global attenuation metric calculated as the fraction of pixels in the attenuation map with an attenuation coefficient greater than a certain threshold $x$, multiplied by 1,000 :

$$
I P A_{x}=\frac{N\left(\mu_{t}>x\right)}{N_{\text {total }}} \times 1000
$$

where the numerator includes pixels with attenuation greater than a certain threshold $x$. For this study, IPA 6 and IPA 11 were calculated. IPA 6 has been associated with fibroatheroma and IPA ${ }_{11}$ is a better discriminator of thin-cap fibroatheroma (TCFA) ${ }^{4}$.

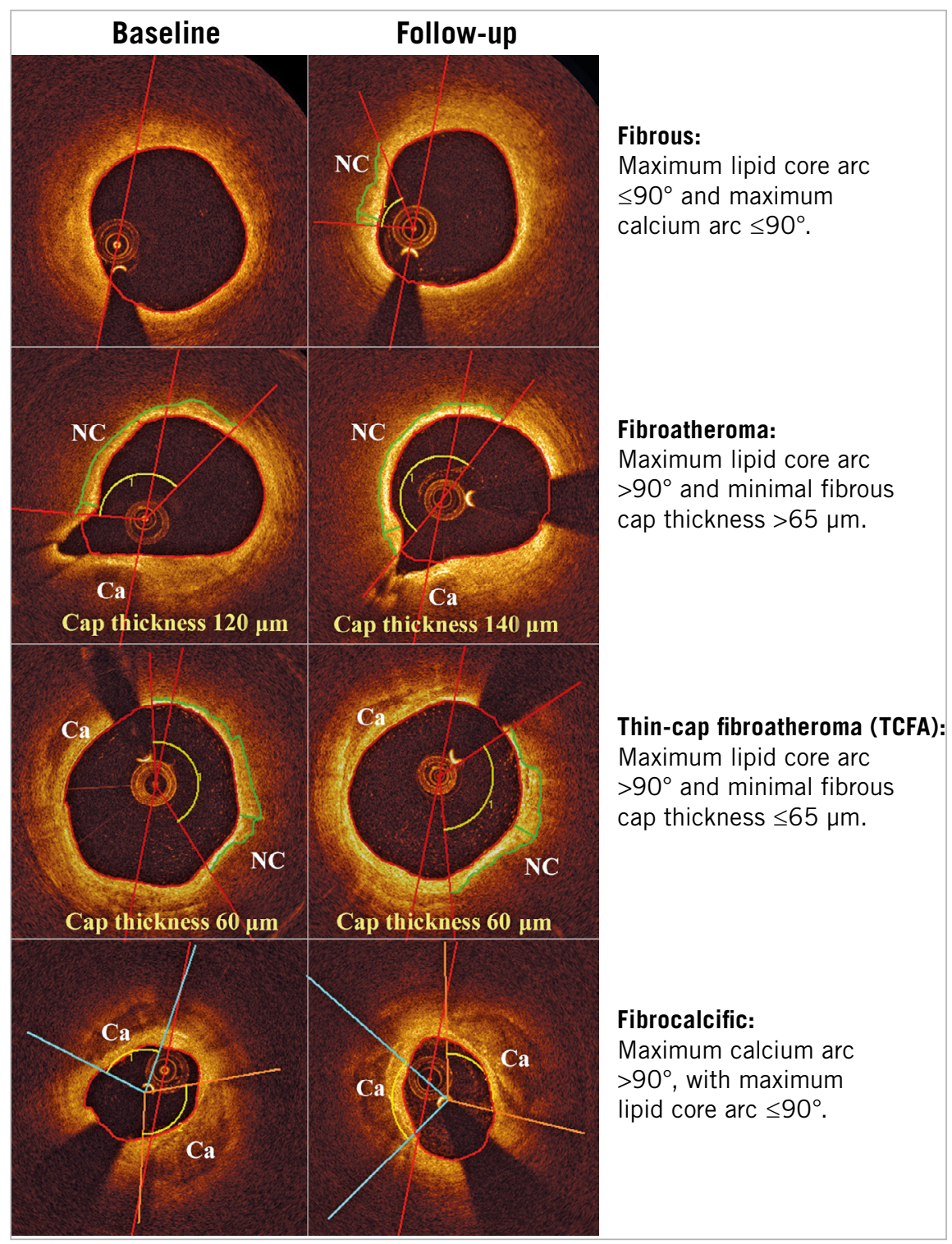

Figure 1. Hierarchical plaque classification. Representative examples of fibrous plaque, fibroatheroma, thin-cap fibroatheroma and fibrocalcific plaque. Red lines denote lipid core localisation, and green lines cap segmentation. Ca: calcium; NC: lipid core 


\section{STATISTICAL ANALYSIS}

All analyses were performed with SPSS, Version 20.0 (IBM Corp., Armonk, NY, USA). Continuous variables are presented as mean \pm SD or median (interquartile range [IQR]), while categorical variables are presented as count (percentage). Mixed model regression for repeated measurements assessed differences in quantitative characteristics between baseline and follow-up (reported as estimates [95\% confidence intervals]), and predictors of changes. Mixed logistic regression for repeated measurements assessed differences in qualitative characteristics and predictors of favourable and unfavourable change in plaque morphology. For segmentlevel analyses, due to the segment clustering within patients, a random intercept adjusted for within-patient correlations. All p-values are two-sided with a value $<0.05$ indicating significance.

\section{Results}

One-hundred and nineteen patients were screened. Thirty-four were excluded due to inadequate length, four due to different branch visualisation, six due to venous graft, two due to poor image quality, and one due to intervention in the studied segment between the two studies. Overall, 72 patients were included.

Baseline clinical characteristics are reported in Table 1. The median follow-up was 6.2 months (IQR 6.0-11.1 months). The follow-up interval did not differ significantly in patients undergoing repeat catheterisation either for follow-up purposes or for recurrent symptoms (median 6 months [IQR 6-9 months] versus 8 months [6-18 months], $\mathrm{p}=0.08$ ). A mean vessel length of $17.83 \pm 9.22 \mathrm{~mm}$ and $17.65 \pm 9.00 \mathrm{~mm}$ was analysed for baseline and follow-up, respectively.

\section{INTER-STUDY VARIABILITY OF OCT MEASUREMENTS}

Inter-study variability was low overall. Based on the analysis, thresholds indicating relevant change were set at $\pm 0.5 \mathrm{~mm}^{2}$ for minimum lumen area (bias: $-0.11 \mathrm{~mm}^{2}$ [95\% limits of agreement: -0.45 to $\left.\left.0.23 \mathrm{~mm}^{2}\right]\right), \pm 1 \mathrm{~mm}^{2}$ for mean lumen area $\left(-0.21 \mathrm{~mm}^{2}\right.$ [-1.03 to $\left.\left.0.61 \mathrm{~mm}^{2}\right]\right), \pm 20^{\circ}$ for lipid core arc (mean: $0.1^{\circ}[-18.2$ to $18.4^{\circ}$; $\max : 1.6^{\circ}\left[-10.5\right.$ to $\left.\left.13.6^{\circ}\right]\right), \pm 7 \%$ for RLCI $(1.0$ [ -5.4 to $7.5]$ ), $\pm 10^{\circ}$ for calcium arc (mean: $-0.3^{\circ}\left[-4.7\right.$ to $\left.4.1^{\circ}\right]$; $\max :-2.2^{\circ}$ [ -18.4 to $\left.\left.14.0^{\circ}\right]\right), \pm 5 \%$ for RCI $(0.5$ [ -1.5 to 2.5$\left.]\right)$, and $\pm 40 \mu \mathrm{m}$ for cap thickness (minimum: $8 \mu \mathrm{m}[-34$ to $50 \mu \mathrm{m}]$; mean: $-5 \mu \mathrm{m}[-39$ to $29 \mu \mathrm{m}]$ ). Table 2 summarises intra- and inter-observer variability.

\section{CHANGES IN OCT MORPHOLOGICAL CHARACTERISTICS}

\section{PATIENT-LEVEL ANALYSIS}

Mean lumen area decreased at follow-up $\left(7.87 \pm 3.68 \mathrm{~mm}^{2}\right.$ versus $8.22 \pm 3.63 \mathrm{~mm}^{2}$ at baseline; $\mathrm{p}=0.049$ ). However, no changes
Table 1. Baseline characteristics.

\begin{tabular}{|c|c|c|}
\hline \multicolumn{2}{|l|}{ Age, years } & $58.98 \pm 10.95$ \\
\hline \multicolumn{2}{|l|}{ Male, n (\%) } & $59(81.9)$ \\
\hline \multicolumn{2}{|l|}{ Interval, months } & $6.2[6.0-11.1]$ \\
\hline \multirow{5}{*}{$\begin{array}{l}\text { Clinical presentation, } \\
\text { baseline, } \mathrm{n}(\%)\end{array}$} & Stable angina & $29(40.3)$ \\
\hline & Unstable angina & $11(15.3)$ \\
\hline & STEMI & $28(38.9)$ \\
\hline & NSTEMI & $3(4.2)$ \\
\hline & Asymptomatic & $1(1.4)$ \\
\hline \multirow{4}{*}{$\begin{array}{l}\text { Clinical presentation, } \\
\text { follow-up, } \mathrm{n}(\%)\end{array}$} & Stable angina & $12(16.7)$ \\
\hline & Unstable angina & $4(5.6)$ \\
\hline & NSTEMI & $1(1.4)$ \\
\hline & Asymptomatic & $55(76.4)$ \\
\hline \multicolumn{2}{|l|}{ Hypertension, n (\%) } & $34(48.6)$ \\
\hline \multicolumn{2}{|l|}{ Diabetes, n (\%) } & $8(11.1)$ \\
\hline \multicolumn{2}{|l|}{ Dyslipidaemia, n (\%) } & $36(52.2)$ \\
\hline \multicolumn{2}{|l|}{ Current smoker, n (\%) } & $27(37.5)$ \\
\hline \multicolumn{2}{|c|}{ Family history of CAD, n (\%) } & $38(53.5)$ \\
\hline \multicolumn{2}{|l|}{ Prior infarction, $\mathrm{n}(\%)$} & $12(16.7)$ \\
\hline \multicolumn{2}{|l|}{ Prior $\mathrm{PCl}, \mathrm{n}(\%)$} & $13(18.1)$ \\
\hline \multicolumn{2}{|l|}{ Prior CABG, n (\%) } & $1(1.4)$ \\
\hline \multicolumn{2}{|c|}{ Chronic kidney disease (eGFR <50 ml/min), $\mathrm{n}(\%)$} & $2(2.8)$ \\
\hline \multirow[t]{3}{*}{ Vessel, n (\%) } & LAD & $38(52.8)$ \\
\hline & $\mathrm{LCx}$ & $20(27.8)$ \\
\hline & RCA & $14(19.4)$ \\
\hline \multirow[t]{5}{*}{ Therapy, n (\%) } & ACE inhibitors/ARBs & $37(51.4)$ \\
\hline & $\begin{array}{l}\text { Moderate-intensity } \\
\text { statins }\end{array}$ & $37(51.4)$ \\
\hline & High-intensity statins & $26(36.1)$ \\
\hline & Beta-blocker & $39(54.2)$ \\
\hline & Thienopyridine & 69 (95.8) \\
\hline \multicolumn{2}{|c|}{ Baseline total cholesterol, $\mathrm{mmol} / \mathrm{L}$} & $5.01 \pm 1.13$ \\
\hline \multicolumn{2}{|l|}{ Baseline LDL-C, mmol/L } & $3.40 \pm 1.10$ \\
\hline \multicolumn{2}{|c|}{ Baseline triglycerides, $\mathrm{mmol} / \mathrm{L}$} & $1.26 \pm 0.90$ \\
\hline \multicolumn{3}{|c|}{$\begin{array}{l}\text { Values expressed as mean } \pm \text { SD, median [interquartile range], or n (\%). } \\
\text { Moderate-intensity statins include simvastatin } 20-40 \mathrm{mg} \text {, atorvastatin } \\
10-20 \mathrm{mg} \text {, or rosuvastatin } 5-10 \mathrm{mg} \text {. High-intensity statins include } \\
\text { atorvastatin } 40-80 \mathrm{mg} \text {, or rosuvastatin } 20-40 \mathrm{mg} \text {. ACE: angiotensin- } \\
\text { converting enzyme; ARB: angiotensin receptor blocker; CABG: coronary } \\
\text { artery bypass graft surgery; CAD: coronary artery disease; } \\
\text { eGFR: estimated glomerular filtration rate; LAD: left anterior descending } \\
\text { artery; LCx: left circumflex; LDL-C: low-density cholesterol; } \\
\text { NSTEMI: non-ST-elevation myocardial infarction; PCI: percutaneous } \\
\text { coronary interventions; RCA: right coronary artery; STEMI: ST-elevation } \\
\text { myocardial infarction }\end{array}$} \\
\hline
\end{tabular}

Table 2. Variability of OCT measurements.

\begin{tabular}{|c|c|c|c|c|}
\hline & Mean lipid core arc, ${ }^{\circ}$ & Mean calcium arc, ${ }^{\circ}$ & Min cap thickness, $\mu \mathrm{m}$ & Mean cap thickness, $\mu \mathrm{m}$ \\
\hline Intra-observer variability & $1.9(-6.8$ to 10.7$)$ & $0.2(-3.4$ to 3.8$)$ & $4(-28$ to 36$)$ & $-3(-39$ to 33$)$ \\
\hline Inter-observer variability & $4.0(-18.9$ to 26.8$)$ & $-1.9(-11.6$ to 7.8$)$ & $2(-31$ to 35$)$ & $10(-43$ to 63$)$ \\
\hline
\end{tabular}


were observed in patient-level plaque morphology. Average but not minimal cap thickness increased over time, while IPA ${ }_{11}$ but not IPA 6 had a borderline decrease $(\mathrm{p}=0.049)$. Relevant changes in lumen dimensions, calcium arc and cap thickness were observed in a few patients (30-35\%); however, $>50 \%$ had relevant differences in maximum lipid core arc, with one third presenting with lipid core increase (Table 3).

\section{SEGMENT-LEVEL ANALYSIS}

Overall, 257 non-culprit segments were assessed. Both minimal and mean lumen area were significantly decreased at follow-up ( $p=0.018$ and $p=0.005$, respectively). There were no significant differences in lipid core or calcium metrics between the two intervals. However, both minimum and average cap thickness were increased at follow-up compared to baseline $(\mathrm{p}=0.019$ and $\mathrm{p}=0.002$, respectively). Additionally, $\mathrm{IPA}_{6}$ and $\mathrm{IPA}_{11}$ at followup were significantly decreased $(p=0.036$ and $p=0.022$, respectively). Relevant changes of lumen dimensions and calcium arc were observed in a few segments $(<40 \%)$, whereas $>50 \%$ had relevant changes in cap thickness and maximum lipid core arc (Table 3).

\section{CHANGES IN HIERARCHICAL PLAQUE MORPHOLOGY}

The changes are summarised in Table 3, Table 4, Figure 2, and Figure 3.

\section{PATIENT-LEVEL ANALYSIS}

In most patients $(58.3 \%)$, plaque morphology did not change. In $25 \%$, plaque morphology had a favourable change (i.e., from TCFA to fibroatheroma, or from fibroatheroma/TCFA to fibrous), with an unfavourable change (i.e., from fibrous to fibroatheroma/ TCFA, or from fibroatheroma to TCFA) in $16.7 \%$. Most fibrous plaques showed no morphological changes $(81 \%)$. Interestingly, $58 \%$ of TCFAs were converted to fibroatheroma, while $30 \%$ of fibroatheromas gave rise to new TCFAs.

\section{SEGMENT-LEVEL ANALYSIS}

No changes were observed in $68.5 \%$ of segments. Favourable change was observed in $18.4 \%$, and unfavourable in $12.9 \%$. Similarly to patient-level analysis, $>80 \%$ of fibrous plaques had no morphological changes, while most TCFAs were converted to fibroatheromas, with new TCFAs emerging from $13 \%$ of baseline fibroatheromas. Consequently, the overall percentage of lesions with a specific morphology did not change over time (Table 4).

\section{PREDICTORS OF CHANGE IN PLAQUE CHARACTERISTICS}

Prediction of change in MLA, maximum lipid core arc, maximum calcium arc, and minimum cap thickness from clinical factors and angiographic variables was examined for all variables of Table 1, but it was poor. Time interval was associated with a decrease in MLA ( $b=-0.04$ [ -0.01 to -0.07$] ; \mathrm{p}=0.004$ ), whereas family history of CAD was associated with an increase $(b=0.40[0.04$ to 0.75]; $p=0.029$ ). From angiographic variables, $\mathrm{LCx}$ lesions had a higher MLA increase compared to RCA lesions $(b=0.57$ [0.02 to 1.12]; $\mathrm{p}=0.043$ ), while location distal to the stented segment was associated with a decrease in maximum lipid core arc compared to
Table 3. Plaque morphology at baseline and follow-up.

\begin{tabular}{|c|c|c|c|c|}
\hline \multicolumn{2}{|c|}{ 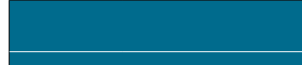 } & Baseline & Follow-up & $p$-value ${ }^{a}$ \\
\hline \multicolumn{5}{|c|}{ Patient level } \\
\hline \multicolumn{2}{|c|}{$\begin{array}{l}\text { Minimal lumen area, } \\
\mathrm{mm}^{2}\end{array}$} & $5.24 \pm 2.86$ & $5.28 \pm 2.93$ & 0.76 \\
\hline \multicolumn{2}{|c|}{ Mean lumen area, $\mathrm{mm}^{2}$} & $8.22 \pm 3.63$ & $7.87 \pm 3.68$ & 0.049 \\
\hline \multirow{4}{*}{$\begin{array}{l}\text { Plaque } \\
\text { morpho- } \\
\text { logy, } \\
\text { n (\%) }\end{array}$} & Fibroatheroma & $30(41.7)$ & $34(47.2)$ & \multirow{4}{*}{0.81} \\
\hline & Fibrocalcific & 0 & 0 & \\
\hline & Fibrous & $16(22.2)$ & $16(22.2)$ & \\
\hline & TCFA & $26(36.1)$ & $22(30.6)$ & \\
\hline \multicolumn{2}{|c|}{ Mean lipid core arc, ${ }^{\circ}$} & $99.9 \pm 35.6$ & $96.7 \pm 35.4$ & 0.21 \\
\hline \multicolumn{2}{|c|}{ Max lipid core arc, ${ }^{\circ}$} & $170.9 \pm 67.4$ & $174.1 \pm 79.7$ & 0.67 \\
\hline \multicolumn{2}{|l|}{$\mathrm{RLCl}, \%$} & $15.24 \pm 10.46$ & $15.18 \pm 10.76$ & 0.91 \\
\hline \multicolumn{2}{|c|}{ Mean calcium arc, ${ }^{\circ}$} & $72.1 \pm 51.0$ & $62.0 \pm 42.8$ & 0.25 \\
\hline \multicolumn{2}{|c|}{ Max calcium arc, ${ }^{\circ}$} & $100.7 \pm 83.8$ & $90.6 \pm 82.9$ & 0.31 \\
\hline \multicolumn{2}{|l|}{$\mathrm{RCl}, \%$} & $1.87 \pm 6.03$ & $1.85 \pm 6.75$ & 0.92 \\
\hline \multicolumn{2}{|c|}{$\begin{array}{l}\text { Minimum cap } \\
\text { thickness, } \mu \mathrm{m}\end{array}$} & $88 \pm 53$ & $91 \pm 65$ & 0.68 \\
\hline \multicolumn{2}{|c|}{$\begin{array}{l}\text { Average cap thickness, } \\
\mu \mathrm{m}\end{array}$} & $183 \pm 62$ & $209 \pm 67$ & 0.01 \\
\hline \multicolumn{2}{|c|}{$\mathrm{IPA}_{6}, \mathrm{~mm}^{-1}$} & $480.71 \pm 144.97$ & $464.36 \pm 152.76$ & 0.48 \\
\hline \multicolumn{2}{|c|}{$\mathrm{IPA}_{11}, \mathrm{~mm}^{-1}$} & $52.40 \pm 41.00$ & $44.73 \pm 36.23$ & 0.049 \\
\hline \multicolumn{5}{|c|}{ Segment level } \\
\hline \multicolumn{2}{|c|}{$\begin{array}{l}\text { Minimal lumen area, } \\
\mathrm{mm}^{2}\end{array}$} & $7.07 \pm 3.75$ & $6.86 \pm 3.73$ & 0.018 \\
\hline \multicolumn{2}{|c|}{ Mean lumen area, $\mathrm{mm}^{2}$} & $8.15 \pm 4.14$ & $7.92 \pm 3.98$ & 0.005 \\
\hline \multirow{4}{*}{$\begin{array}{l}\text { Plaque } \\
\text { morpho- } \\
\text { logy, } \\
\text { n (\%) }\end{array}$} & Fibroatheroma & $105(40.9)$ & $110(42.8)$ & \multirow{4}{*}{0.84} \\
\hline & Fibrocalcific & $1(0.4)$ & 0 & \\
\hline & Fibrous & $118(45.9)$ & $123(47.9)$ & \\
\hline & TCFA & $33(12.8)$ & $24(9.3)$ & \\
\hline \multicolumn{2}{|c|}{ Mean lipid core arc, ${ }^{\circ}$} & $96.7 \pm 43.0$ & $94.8 \pm 39.1$ & 0.48 \\
\hline \multicolumn{2}{|c|}{ Max lipid core arc, ${ }^{\circ}$} & $133.7 \pm 65.0$ & $135.4 \pm 71.0$ & 0.81 \\
\hline \multicolumn{2}{|l|}{$\mathrm{RLCl}, \%$} & $15.33 \pm 15.07$ & $15.15 \pm 14.27$ & 0.76 \\
\hline \multicolumn{2}{|c|}{ Mean calcium arc, ${ }^{\circ}$} & $76.9 \pm 59.9$ & $82.5 \pm 56.4$ & 0.59 \\
\hline \multicolumn{2}{|c|}{ Max calcium arc, ${ }^{\circ}$} & $98.4 \pm 83.9$ & $108.6 \pm 89.5$ & 0.34 \\
\hline \multicolumn{2}{|l|}{$\mathrm{RCl}, \%$} & $1.59 \pm 6.11$ & $1.79 \pm 7.03$ & 0.38 \\
\hline \multicolumn{2}{|c|}{$\begin{array}{l}\text { Minimum cap } \\
\text { thickness, } \mu \mathrm{m}\end{array}$} & $120 \pm 75$ & $134 \pm 81$ & 0.019 \\
\hline \multicolumn{2}{|c|}{$\begin{array}{l}\text { Average cap thickness, } \\
\mu \mathrm{m}\end{array}$} & $184 \pm 80$ & $206 \pm 83$ & 0.002 \\
\hline $\mathrm{IPA}_{6}, \mathrm{mn}$ & & $472.77 \pm 179.94$ & $444.52 \pm 185.86$ & 0.036 \\
\hline $\mathrm{IPA}_{11}, \mathrm{~m}$ & $m^{-1}$ & $49.46 \pm 52.42$ & $42.72 \pm 45.74$ & 0.022 \\
\hline
\end{tabular}

Values expressed as mean $\pm \mathrm{SD}$, or $\mathrm{n}(\%)$. a $p$-values derived from mixed model regression for repeated measurements. IPA: index of plaque attenuation; $\mathrm{RCl}$ : relative calcium index; $\mathrm{RLCl}$ : relative lipid core index

proximally located lesions $(b=-20.27$ [ -38.06 to -2.48$] ; p=0.026)$. Baseline morphology was also not predictive of changes in these variables.

Predictors of favourable or unfavourable change in plaque morphology were examined, with the only significant negative 


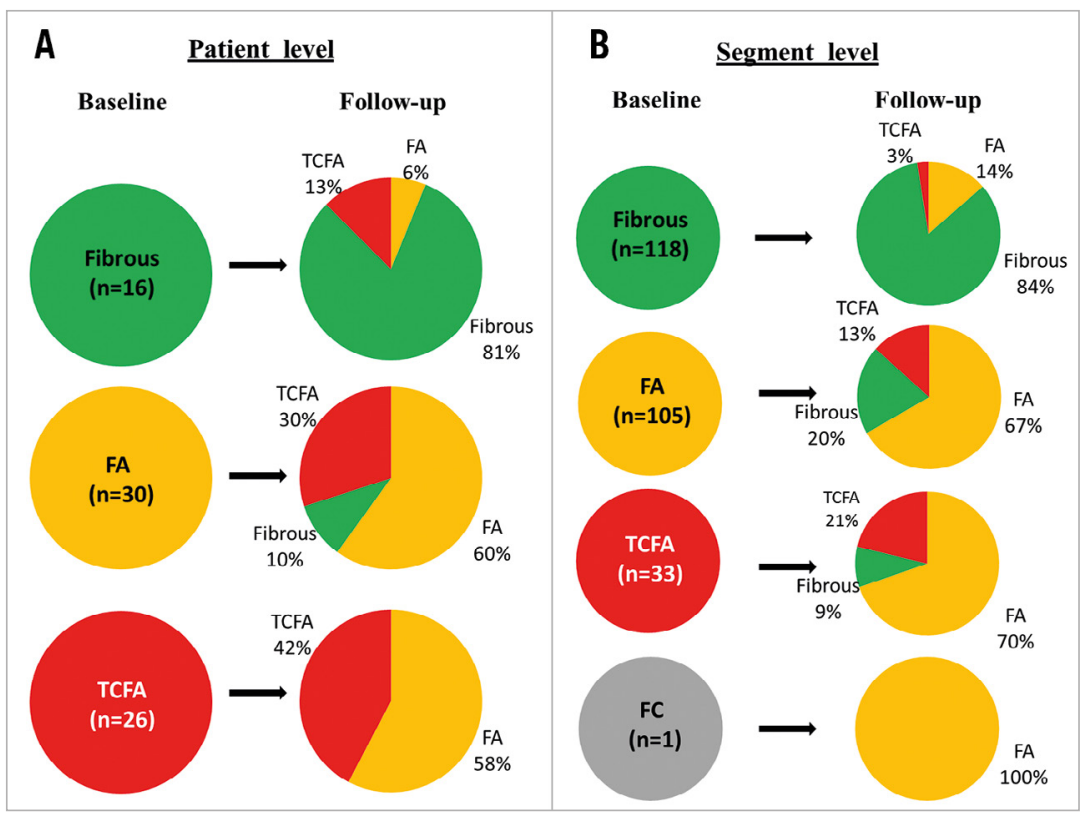

Figure 2. Changes in hierarchical plaque morphology. A) Patient level. B) Segment level. FA: fibroatheroma; FC: fibrocalcific; TCFA: thin-cap fibroatheroma

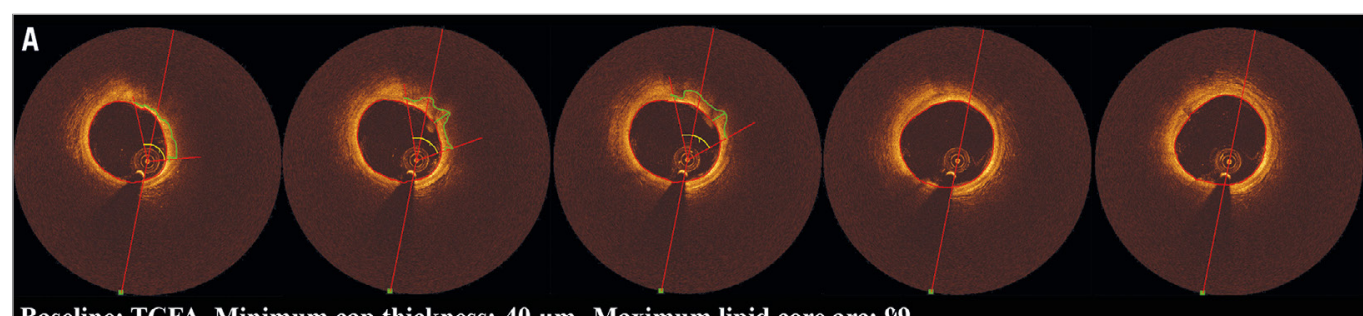

Baseline: TCFA. Minimum cap thickness: $40 \mu \mathrm{m}$. Maximum lipid core arc: 99

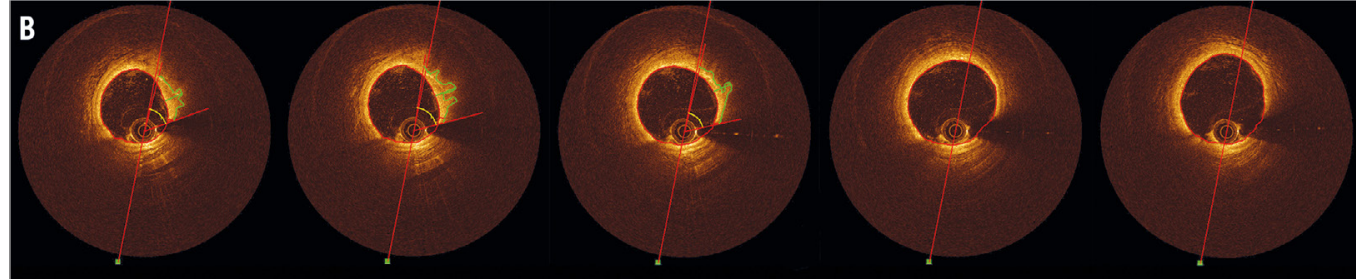

Follow-up: Fibrous. Minimum cap thickness: 70 mm. Maximum lipid core arc: 85

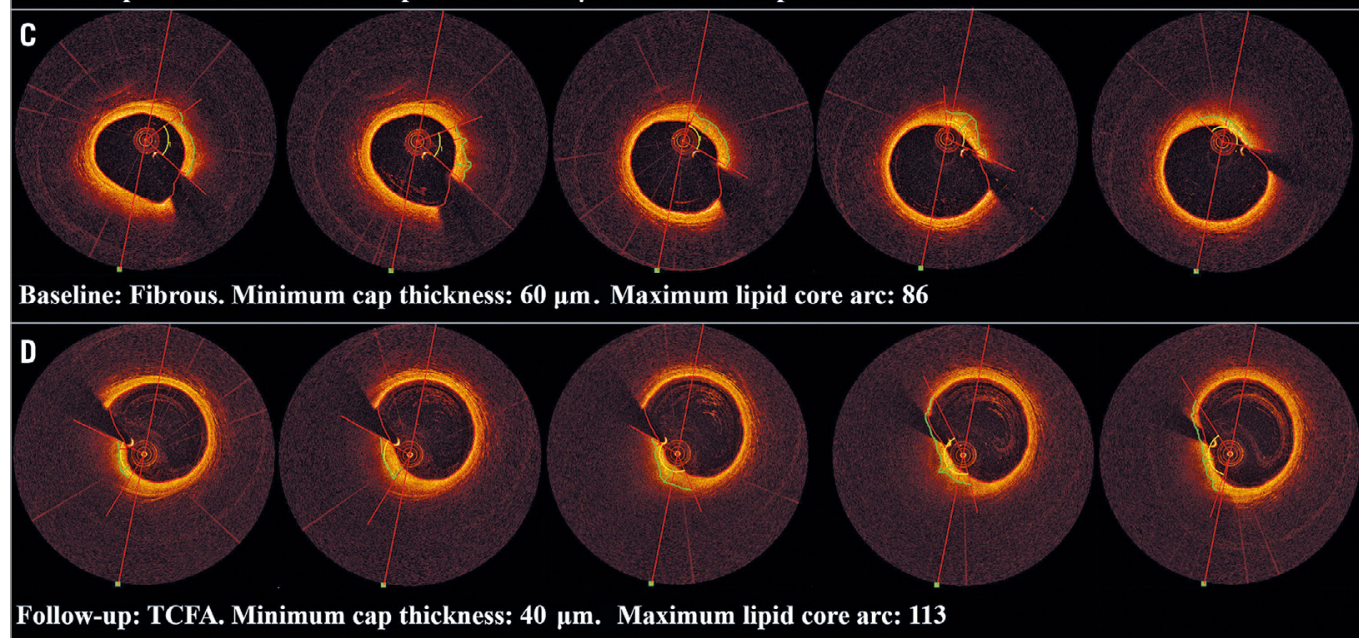

Figure 3. Examples of segment-level change. A) \& B) From TCFA to fibrous. C) \& D) From fibrous to TCFA. 
Table 4. Changes in OCT characteristics over time.

\begin{tabular}{|c|c|c|}
\hline & Patient level $(n=72)$ & $\begin{array}{l}\text { Segment level } \\
(n=257)\end{array}$ \\
\hline \multicolumn{3}{|l|}{ MLA } \\
\hline $\begin{array}{l}\text { Difference (follow-up- } \\
\text { baseline), } \mathrm{mm}^{2}\end{array}$ & $\begin{array}{c}0.05(-0.26 \text { to } 0.35) \\
p=0.76\end{array}$ & $\begin{array}{c}-0.22(-0.39 \text { to }-0.04) \\
p=0.018\end{array}$ \\
\hline $\begin{array}{l}\text { Lumen expansion, } \\
\mathrm{n}(\%)\end{array}$ & $12(16.7)$ & $35(13.6)$ \\
\hline No change, $\mathrm{n}(\%)$ & $47(65.3)$ & $158(61.5)$ \\
\hline $\begin{array}{l}\text { Lumen narrowing, } \\
\mathrm{n}(\%)\end{array}$ & $13(18.1)$ & $64(24.9)$ \\
\hline \multicolumn{3}{|l|}{ Max lipid core arc } \\
\hline $\begin{array}{l}\text { Difference (follow-up- } \\
\text { baseline), }^{\circ}\end{array}$ & $\begin{array}{c}3.4(-11.5 \text { to } 18.4) \\
p=0.65\end{array}$ & $\begin{array}{c}1.0(-7.2 \text { to } 9.2) \\
p=0.81\end{array}$ \\
\hline Increase, n (\%) & $15(20.8)$ & $67(26.1)$ \\
\hline No change, n (\%) & $33(45.8)$ & $124(48.2)$ \\
\hline Decrease, n (\%) & $24(33.3)$ & $66(25.7)$ \\
\hline \multicolumn{3}{|l|}{ Max calcium arc } \\
\hline $\begin{array}{l}\text { Difference (follow-up- } \\
\text { baseline), }^{\circ}\end{array}$ & $\begin{array}{c}-10.8(-30.3 \text { to } 8.7) \\
p=0.27\end{array}$ & $\begin{array}{c}7.2(-8.0 \text { to } 22.4) \\
p=0.34\end{array}$ \\
\hline Increase, n (\%) & $10(13.9)$ & $32(12.5)$ \\
\hline No change, $\mathrm{n}(\%)$ & $50(69.4)$ & $202(78.6)$ \\
\hline Decrease, n (\%) & $12(16.7)$ & $23(8.9)$ \\
\hline \multicolumn{3}{|l|}{ Minimum cap thickness } \\
\hline $\begin{array}{l}\text { Difference (follow-up- } \\
\text { baseline), } \mu \mathrm{m}\end{array}$ & $\begin{array}{c}6(-12 \text { to } 24) \\
p=0.39\end{array}$ & $\begin{array}{c}16 \text { (2 to } 29) \\
p=0.019\end{array}$ \\
\hline Increase, n (\%) & 12 (19.0) & $52(31.0)$ \\
\hline No change, $\mathrm{n}(\%)$ & $40(63.5)$ & $81(48.2)$ \\
\hline Decrease, n (\%) & $11(17.5)$ & $35(20.8)$ \\
\hline \multicolumn{3}{|c|}{ Hierarchical morphology } \\
\hline $\begin{array}{l}\text { Favourable change, } \\
\mathrm{n}(\%)\end{array}$ & $18(25.0)$ & $47(18.3)$ \\
\hline FA $\rightarrow$ Fibrous & $3(4.2)$ & $21(8.2)$ \\
\hline TCFA $\rightarrow$ Fibrous & $0(0)$ & $3(1.2)$ \\
\hline TCFA $\rightarrow$ FA & $15(20.8)$ & $23(8.9)$ \\
\hline No change, $\mathrm{n}(\%)$ & $42(58.3)$ & $176(68.5)$ \\
\hline Fibrous $\rightarrow$ Fibrous & $13(18.1)$ & $99(38.5)$ \\
\hline $\mathrm{FA} \rightarrow \mathrm{FA}$ & $18(25.0)$ & $70(27.2)$ \\
\hline TCFA $\rightarrow$ TCFA & $11(15.3)$ & $7(2.7)$ \\
\hline $\begin{array}{l}\text { Unfavourable change, } \\
\text { n (\%) }\end{array}$ & $12(16.7)$ & $33(12.8)$ \\
\hline TCFA $\rightarrow F A$ & $9(12.5)$ & $14(5.4)$ \\
\hline Fibrous $\rightarrow F A$ & $1(1.4)$ & $16(6.2)$ \\
\hline Fibrous $\rightarrow$ TCFA & $2(2.8)$ & $3(1.2)$ \\
\hline \multicolumn{3}{|c|}{$\begin{array}{l}\text { Values expressed as estimate (95\% confidence interval), or } \mathrm{n}(\%) \text {. } \\
\text { FA: fibroatheroma; FC: fibrocalcific; MLA: minimal lumen area; } \\
\text { TCFA: thin-cap fibroatheroma }\end{array}$} \\
\hline
\end{tabular}

predictor of favourable change being family history of CAD (OR: 0.383 [0.188-0.781]; $\mathrm{p}=0.009)$. Lesion location in the LAD or LCx predicted less frequent favourable change and more frequent unfavourable change compared to the RCA, albeit not reaching significance.

\section{ATTENUATION ANALYSIS AND PLAQUE MORPHOLOGY}

Attenuation patient- and segment-level analyses per plaque type are presented in Table 5. In both analyses, IPA 6 and IPA 11 differed significantly between plaque types, with values of both attenuation coefficients higher for TCFA compared to fibroatheroma or fibrous, and higher for fibroatheroma compared to fibrous (all p-values $<0.05$ ). Both indices had a strong positive correlation with lipid core metrics and a strong negative correlation with cap thickness $(\mathrm{p}<0.001)$, also after adjustment for the time interval.

\section{Table 5. Association of plaque classification with attenuation.}

\begin{tabular}{|c|c|c|c|c|}
\hline $\begin{array}{l}\text { Plaque } \\
\text { morphology }\end{array}$ & Value & $\begin{array}{l}p \text {-value } \\
\text { vs. FA }\end{array}$ & $\begin{array}{l}p \text {-value } \\
\text { vs. FC }\end{array}$ & $\begin{array}{l}p \text {-value } \\
\text { vs. fibrous }\end{array}$ \\
\hline \multicolumn{5}{|l|}{ Patient level } \\
\hline \multicolumn{5}{|l|}{$\mathrm{IPA}_{6}, \mathrm{~mm}^{-1}$} \\
\hline TCFA & $533.6 \pm 134.7$ & 0.019 & & $<0.001$ \\
\hline FA & $485.2 \pm 122.4$ & & & 0.015 \\
\hline Fibrous & $364.5 \pm 156.4$ & & & \\
\hline \multicolumn{5}{|l|}{$\mathrm{IPA}_{11}, \mathrm{~mm}^{-1}$} \\
\hline TCFA & $66.9 \pm 45.6$ & 0.018 & & $<0.001$ \\
\hline FA & $48.7 \pm 31.6$ & & & 0.006 \\
\hline Fibrous & $17.6 \pm 14.8$ & & & \\
\hline \multicolumn{5}{|l|}{ Segment level } \\
\hline \multicolumn{5}{|l|}{$\mathrm{IPA}_{6}, \mathrm{~mm}^{-1}$} \\
\hline TCFA & $605.3 \pm 127.7$ & 0.013 & 0.163 & $<0.001$ \\
\hline FA & $516.4 \pm 143.5$ & & 0.315 & $<0.001$ \\
\hline $\mathrm{FC}$ & 398.1 & & & 0.903 \\
\hline Fibrous & $359.6 \pm 189.6$ & & & \\
\hline \multicolumn{5}{|l|}{$\mathrm{IPA}_{11}, \mathrm{~mm}^{-1}$} \\
\hline TCFA & $89.5 \pm 66.5$ & 0.002 & 0.601 & $<0.001$ \\
\hline FA & $53.4 \pm 46.7$ & & 0.891 & $<0.001$ \\
\hline $\mathrm{FC}$ & 14.0 & & & 0.827 \\
\hline Fibrous & $30.0 \pm 60.7$ & & & \\
\hline \multicolumn{5}{|c|}{$\begin{array}{l}\text { Values expressed as mean } \pm \text { SD. } p \text {-values derived from mixed model } \\
\text { regression. FA: fibroatheroma; FC: fibrocalcific; IPA: index of plaque } \\
\text { attenuation; TCFA: thin-cap fibroatheroma }\end{array}$} \\
\hline
\end{tabular}

\section{EXAMPLE OF CLINICALLY RELEVANT MORPHOLOGICAL CHANGE}

There was only one patient with a new clinical syndrome attributed to lesion progression (Figure 4). This patient who had undergone stent implantation in the RCA due to NSTEMI presented with stable angina 20 months later due to progression of a moderate mid LAD lesion. OCT revealed progressive lumen narrowing in a fibroatheroma, distal to a segment with several sites of plaque rupture. Interestingly, in the same patient there was lipid core reduction and lumen expansion in a proximal lesion.

\section{Discussion}

In the current study, we performed comprehensive patient-level and segment-level OCT analyses of non-culprit plaque morphology, 

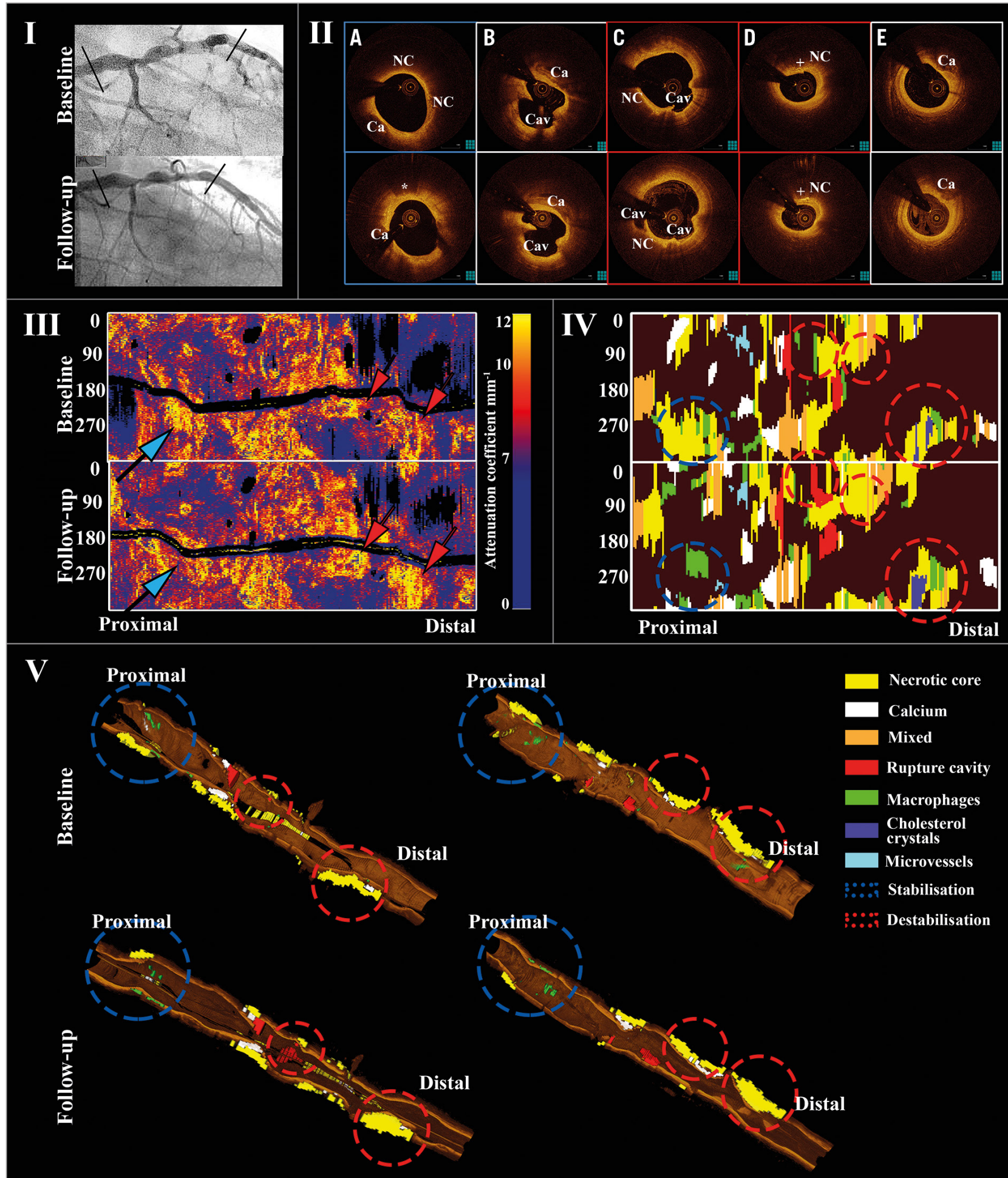

Figure 4. Example of clinically evident non-culprit lesion progression. I. Angiogram of LAD in a person presenting with new anginal symptoms 20 months after PCI of LCX for unstable angina. II. Cross-sectional OCT showing progressive narrowing at a fibroatheroma distally (D). Mid segment, two plaque rupture sites at baseline, with development of third rupture site at follow-up (B \& C). Proximally, fibroatheroma with lipid core regression at follow-up (A). E) Distal segment with small calcification and no significant morphological or luminal differences. *macrophages. + cholesterol crystals. Ca: calcium; Cav: cavity; NC: lipid core. III. Attenuation spread-out maps showing the longitudinal distribution of maximum attenuation coefficient values along the studied segment at baseline and follow-up. One site proximally had a reduction (blue arrow), and two mid and distal sites an increase (red arrow). IV. Plaque spread-out maps with colour-coded tissues demonstrating lipid core reduction proximally (blue circles), a new rupture at the mid segment and lipid core increase distally (red circles). $V$. Three-dimensional views demonstrating these morphological changes (QAngioOCT 3D; Medis Specials bv, Leiden, the Netherlands). 
for evaluating dynamic changes of atherosclerosis under standard medical treatment. To our knowledge, this is the first study to have used systematic OCT analysis over multiple coronary segments with meticulous matching, for assessing morphological changes over time. Quantitative attenuation analysis further corroborated findings from conventional plaque characterisation. Our main findings were that, in patients with CAD: 1) change in nonculprit plaque morphology over time occurs frequently, namely in $42 \%$ of patients and $31 \%$ of segments; 2 ) there are large differences between individual patients and segments with both favourable and unfavourable changes; 3) the frequent morphological changes are unapparent when averaged in the cohort; 4) a modest fibrous cap thickening is observed in individual segments, leading to increase of patient-level average cap thickness, but not of the minimal cap thickness; 5) these changes are rarely accompanied by clinical manifestations; and 6) prediction of these changes from clinical factors and based on initial morphology is poor.

\section{CHANGES OF NON-CULPRIT PLAQUE MORPHOLOGY OVER TIME}

Although pathologic studies have demonstrated a pivotal role for plaque morphology in ACS pathogenesis ${ }^{1}$, the processes underlying changes in plaque morphology in vivo are poorly understood. A previous OCT study with single-frame analysis demonstrated lesion progression and regression over 12 months at a similar incidence of $\sim 30 \%$, and no significant change in $\sim 40 \%$. Interestingly, plaque stabilisation with reduction of TCFA incidence was observed regardless of luminal changes ${ }^{12}$. In our study, at a median of six months, plaque phenotype changed in $42 \%$ of patients and $31 \%$ of non-culprit segments, which was more commonly observed in TCFAs and fibroatheromas. Although fibrous plaques did not change as often, due to their high prevalence the absolute number of fibrous plaques with unfavourable change was higher than the corresponding number of fibroatheromas, showing that all plaque types are prone to favourable or unfavourable changes.

\section{CHANGE IN FIBROUS CAP THICKNESS}

Fibrous cap thickness is a major determinant of plaque vulnerability $^{1}$. In our study, minimum and average segment-level cap thickness increased from baseline to follow-up by 15-20 $\mu \mathrm{m}$. Similarly, an increase in cap thickness over short segments has been demonstrated after statin administration ${ }^{13}$. The increase in segment-level minimum cap thickness in our study led to an increase in patientlevel average cap thickness, but not patient-level minimum cap thickness. Thus, the focal instability per patient as expressed by patient-level cap thickness remained the same, despite a favourable effect on average on the sum of the plaques. Accordingly, a non-negligible percentage of segments $(21 \%)$ had fibrous cap thinning. This suggests that, although statin treatment might promote plaque stabilisation on a population level and even on a coronary tree level, this effect is not observed in all patients and in all individual segments, with observations of cap thinning in specific patients or segments despite intensive systematic therapy. This might call for additional or alternative (eventually local) therapeutic concepts and a tailored approach.

\section{CLINICAL RELEVANCE}

Previous studies have demonstrated an association of non-culprit plaque morphology by virtual histology intravascular ultrasound (VH-IVUS) with future adverse coronary events ${ }^{2}$. Yet, this association was only modest, considering the low incidence of adverse events and a high prevalence of VH-TCFA (40-60\%), and could be largely explained by limitations of VH-IVUS in detection of lipid core and thin fibrous cap ${ }^{14,15}$. Our study underscores another important limitation of intracoronary imaging in identifying highrisk plaques. Even with high-resolution imaging, such as $\mathrm{OCT}^{15}$, we demonstrated that plaque morphology on its own is subject to dynamic changes that might occur without clinical manifestations, and therefore its potential use as a prognostic tool is questionable. Even in our observation of clinically relevant progression due to progressive luminal narrowing, plaque regression was observed in another segment, supporting the hypothesis that morphological plaque changes are highly localised and observed on plaque level rather than patient level. Moreover, these morphological changes were poorly predicted by patient-level or lesion-level factors, implying a limited ability to predict lesion behaviour, possibly due to the effect of local haemodynamics on plaque morphology, with low wall shear stress being associated with reduction of luminal dimensions and development of high-risk plaque morphology ${ }^{16}$.

\section{AUTOMATED PLAQUE CHARACTERISATION}

Increased attenuation has been associated with lipid core and macrophages ${ }^{10}$. Attenuation analysis was in line with conventional OCT plaque characterisation showing increasing values of attenuation indices from fibrous to fibroatheroma to TCFA, corroborating our findings. Additionally, both segment-level IPA 6 and IPA decreased over time, paralleling the increase in fibrous cap thickness. This finding together with the strong correlations of these indices with increased lipid core and reduced cap thickness suggests that this analysis could be employed for monitoring changes in plaque morphology over time.

\section{Limitations}

This is a small retrospective single-centre registry, and thus prone to the limitations of all retrospective, non-randomised studies. In order to account for the small sample, a comprehensive systematic analysis of plaque morphology was performed and further corroborated by quantitative analysis. Furthermore, using meticulously matched subsegments allowed us to study morphological changes in numerous segments and detect within-patient differences, not detectable by patient-level analysis. As an imaging registry, minor differences in patient characteristics compared to all patients undergoing catheterisation might exist ${ }^{5}$. As OCT lacks tissue penetration, it cannot elucidate mechanisms of luminal loss, which studies with a combination of intravascular ultrasound and OCT 
might reveal. Moreover, the absence of a pre-specified medical regimen does not allow generalisation of our findings. Although PCI could have modified non-culprit plaque morphology, excluding $5 \mathrm{~mm}$ stent edges minimises this possibility, while the finding of favourable or unfavourable change was the same in stented and non-stented arteries. Due to the limited patient sample size and the short follow-up, our study was underpowered for identifying an association between morphological changes and clinical events. Although the short follow-up hampers the long-term evaluation of the dynamics of plaque morphology, it demonstrates that changes are frequently observed over short time periods ${ }^{13,16}$.

\section{Conclusions}

In non-culprit coronary segments of patients with CAD under standard medical therapy, fibrous cap thickness increases over time, resulting in an increase of patient-level average cap thickness, whereas patient-level minimal cap thickness does not change. Moreover, morphological changes over time in individual segments, both favourable and unfavourable, are frequent and highly heterogeneous.

\section{Impact on daily practice}

Our findings add to the current understanding of progression of non-culprit segment atherosclerosis in vivo under standard medical therapy. The finding of dynamic changes occurring even in different segments of the same patient underscores the limitations of current imaging methods in detection of highrisk plaques ${ }^{15}$, and a need for identification of additional markers of plaque instability ${ }^{17}$. Consequently, this calls for new or hybrid imaging modalities, that can better discriminate in vivo progression-prone plaques associated with increased risk for future events.

\section{Funding}

The Thoraxcenter has received research grants from St. Jude Medical and Terumo Europe.

\section{Conflict of interest statement}

B.C. Zhang received financial support from China Postdoctoral Science Foundation Research Funds (Grant: 2013M540468), The Natural Science Foundation of Jiangsu Province (Grant: BK20141137), National Natural Science Foundation of China (Grant: 81670393), China Scholarship Council Fellowship (Grant: 201308310403), Jiangsu Provincial Medical Youth Talent (Grant No: QNRC2016779), and The Science and Technology Department of Xuzhou City (Grant No: KC16SY158). A. Karanasos and J. van der Sijde received research support from St. Jude Medical outside the submitted work. R.J. van Geuns received speaker's fees from Abbott Vascular. The Erasmus MC has a patent licensing agreement with Terumo Corporation and G. van Soest may receive royalties as part of this agreement. The other authors have no conflicts of interest to declare.

\section{References}

1. Falk E, Nakano M, Bentzon JF, Finn AV, Virmani R. Update on acute coronary syndromes: the pathologists' view. Eur Heart J. 2013;34:719-28.

2. Stone GW, Maehara A, Lansky AJ, de Bruyne B, Cristea E, Mintz GS, Mehran R, McPherson J, Farhat N, Marso SP, Parise H, Templin B, White R, Zhang Z, Serruys PW; PROSPECT Investigators. A prospective natural-history study of coronary atherosclerosis. N Engl J Med. 2011;364:226-35.

3. Karanasos A, Ligthart J, Witberg K, van Soest G, Bruining N, Regar E. Optical Coherence Tomography: Potential Clinical Applications. Curr Cardiovasc Imaging Rep. 2012;5:206-20.

4. Gnanadesigan M, Kameyama T, Karanasos A, van Ditzhuijzen NS, van der Sijde JN, van Geuns RJ, Ligthart J, Witberg K, Ughi GJ, van der Steen AF, Regar E, van Soest G. Automated characterisation of lipid core plaques in vivo by quantitative optical coherence tomography tissue type imaging. EuroIntervention. 2016;12:1490-7.

5. van der Sijde JN, Karanasos A, van Ditzhuijzen NS, Okamura T, van Geuns RJ, Valgimigli M, Ligthart JM, Witberg KT, Wemelsfelder S, Fam JM, Zhang B, Diletti R, de Jaegere PP, van Mieghem NM, van Soest G, Zijlstra F, van Domburg RT, Regar E. Safety of optical coherence tomography in daily practice: a comparison with intravascular ultrasound. Eur Heart $J$ Cardiovasc Imaging. 2017;18:467-74.

6. van Ditzhuijzen NS, Karanasos A, Bruining N, van den Heuvel M, Sorop O, Ligthart J, Witberg K, Garcia-Garcia HM, Zijlstra F, Duncker DJ, van Beusekom HM, Regar E. The impact of Fourier-Domain optical coherence tomography catheter induced motion artefacts on quantitative measurements of a PLLA-based bioresorbable scaffold. Int $J$ Cardiovasc Imaging. 2014;30: 1013-26.

7. Jang IK, Tearney GJ, MacNeill B, Takano M, Moselewski F, Iftima N, Shishkov M, Houser S, Aretz HT, Halpern EF, Bouma BE. In vivo characterization of coronary atherosclerotic plaque by use of optical coherence tomography. Circulation. 2005;111:1551-5.

8. Radu MD, Yamaji K, Garcia-Garcia HM, Zaugg S, Taniwaki M, Koskinas KC, Serruys PW, Windecker S, Dijkstra J, Räber L. Variability in the measurement of minimum fibrous cap thickness and reproducibility of fibroatheroma classification by optical coherence tomography using manual versus semi-automatic assessment. EuroIntervention. 2016;12:e987-97.

9. Jamil Z, Tearney G, Bruining N, Sihan K, van Soest G, Ligthart J, van Domburg R, Bouma B, Regar E. Interstudy reproducibility of the second generation, Fourier domain optical coherence tomography in patients with coronary artery disease and comparison with intravascular ultrasound: a study applying automated contour detection. Int J Cardiovasc Imaging. 2013;29:39-51.

10. van Soest G, Goderie T, Regar E, Koljenovic S, van Leenders GL, Gonzalo N, van Noorden S, Okamura T, Bouma BE, Tearney GJ, Oosterhuis JW, Serruys PW, van der Steen AF. Atherosclerotic tissue characterization in vivo by optical coherence tomography attenuation imaging. J Biomed Opt. 2010;15:011105. 
11. Karanasos A, Simsek C, Gnanadesigan M, van Ditzhuijzen NS, Freire R, Dijkstra J, Tu S, Van Mieghem N, van Soest G, de Jaegere P, Serruys PW, Zijlstra F, van Geuns RJ, Regar E. OCT assessment of the long-term vascular healing response 5 years after everolimus-eluting bioresorbable vascular scaffold. $\mathrm{J} \mathrm{Am} \mathrm{Coll}$ Cardiol. 2014;64:2343-56.

12. Xie Z, Tian J, Ma L, Du H, Dong N, Hou J, He J, Dai J, Liu X, Pan H, Liu Y, Yu B. Comparison of optical coherence tomography and intravascular ultrasound for evaluation of coronary lipid-rich atherosclerotic plaque progression and regression. Eur Heart $J$ Cardiovasc Imaging. 2015;16:1374-80.

13. Komukai K, Kubo T, Kitabata H, Matsuo Y, Ozaki Y, Takarada S, Okumoto Y, Shiono Y, Orii M, Shimamura K, Ueno S, Yamano T, Tanimoto T, Ino Y, Yamaguchi T, Kumiko H, Tanaka A, Imanishi T, Akagi H, Akasaka T. Effect of atorvastatin therapy on fibrous cap thickness in coronary atherosclerotic plaque as assessed by optical coherence tomography: the EASY-FIT study. J Am Coll Cardiol. 2014;64:2207-17.

14. Pu J, Mintz GS, Brilakis ES, Banerjee S, Abdel-Karim AR, Maini B, Biro S, Lee JB, Stone GW, Weisz G, Maehara A. In vivo characterization of coronary plaques: novel findings from compar- ing greyscale and virtual histology intravascular ultrasound and near-infrared spectroscopy. Eur Heart J. 2012;33:372-83.

15. Brown AJ, Obaid DR, Costopoulos C, Parker RA, Calvert PA, Teng Z, Hoole SP, West NE, Goddard M, Bennett MR. Direct Comparison of Virtual-Histology Intravascular Ultrasound and Optical Coherence Tomography Imaging for Identification of Thin-Cap Fibroatheroma. Circ Cardiovasc Imaging. 2015;8: e003487.

16. Stone PH, Saito S, Takahashi S, Makita Y, Nakamura S, Kawasaki T, Takahashi A, Katsuki T, Nakamura S, Namiki A, Hirohata A, Matsumura T, Yamazaki S, Yokoi H, Tanaka S, Otsuji S, Yoshimachi F, Honye J, Harwood D, Reitman M, Coskun AU, Papafaklis MI, Feldman CL; PREDICTION Investigators. Prediction of progression of coronary artery disease and clinical outcomes using vascular profiling of endothelial shear stress and arterial plaque characteristics: the PREDICTION study. Circulation. 2012;126:172-81.

17. Toutouzas K, Benetos G, Karanasos A, Chatzizisis YS, Giannopoulos AA, Tousoulis D. Vulnerable plaque imaging: updates on new pathobiological mechanisms. Eur Heart J. 2015;36: 3147-54. 